\title{
On Bilirubin-metal Complex Compounds in Relation to Black Pigments of Gallstones
}

\author{
Noriyoshi Suzuki \\ Department of Surgery (Prof. T. Maki), \\ Tohoku University School of Medicine, Sendai
}

\begin{abstract}
Gallstones occasionally contain black pigments which consist mostly of polymers of bilirubin derivatives. In order to study whether any bile pigmentmetal complex is also concerned with such black pigments of the gallstone, attempts were made at synthesis of bilirubin-Cu complexes. When free bilirubin and cupric chloride were mixed in a solvent mixture of chloroform and ethanol, a dark blue or black substance was formed. This pigment was soluble in ethanol and showed a characteristic absorption at $350 \mathrm{~m} \mu$ (Soret band) and two visible absorption bands at $595 \mathrm{~m} \mu$ and $645 \mathrm{~m} \mu$. Infrared spectroscopy has revealed that this compound is a complex salt of bilimubin and copper having an analogous structure to metalloporphyrins, in which bilirubin has a ring structure of tetrapyrroles and copper is located in the center of nitrogen atoms of pyrroles. On the other hand, addition of cupric chloride to bilirubin in sodium hydroxide solution resulted in sedimentation of a black substance. This pigment has proved spectrometrically to be a bilirubin- $\mathrm{Na}-\mathrm{Cu}$ complex formed by coordination of copper to sodium bilirubinate. Thus, it seems to be possible that the black pigments of the gallstone include some metal-complexes of bile pigments.
\end{abstract}

There is an unusual variety of the gallstone which is characterized by black appearance of the surface and transections. My previous experiments revealed that black pigments were contained in such gallstones which differed from melanins $^{1}$ and consisted mainly of polymers of bilirubin derivatives. ${ }^{2}$ However, considering the presence of copper ${ }^{3}$ and other metal elements in bile at significant concentrations, it is also possible that metal complexes of bile pigments, e.g., bilirubin-copper complexes, are produced in vivo and incorporated into the gallstone, and take part in its black coloration. To evaluate this possibility on experimental basis, a bilirubin-copper complex and a bilirubin-sodium-copper complex, being dark blue or black in color, were prepared in vitro and their structures and general properties studied by spectrometric methods.

Received for publication, July 18, 1966. 


\section{Methods}

Outline of methods for preparation of bilirubin complexes

Bilirubin-Cu complex: This complex compound was produced when a chloroform solution of free dibasic acid bilirubin (I) and an ethanol solution of cupric chloride (II) were mixed and the mixture (III) allowed to stand at room temperature. The color of the mixture III ranged between green and blue according to the mole ratio of bilirubin vs. $\mathrm{Cu}$ and also to that of chloroform vs. ethanol. In order to prevent oxidative denaturation of the complex compound by the effect of excess $\mathrm{Cu}^{++}$, the solution III had to be freed from unreacting $\mathrm{Cu}^{++}$. For this purpose, the solution III was transferred into a separation funnel and washed with distilled water repeatedly, supplementing a small portion of ethanol to the solution before every step of the washing. The potassium ethylxanthogenate test was utilized to detect $\mathrm{Cu}^{++}$in the washings. The washed solution (IV) freed from $\mathrm{Cu}^{++}$was evaporated up and dried in nitrogen flow. The residual solid material (V) was extracted with chloroform and then the residue (VI) extracted with ethanol. Finally, the ethanol extract (VII) was evaporated and dried up in nitrogen flow, and the objective complex compound was recovered as a solid substance (VIII).

Bilirubin-Na-Cu complex: Free dibasic acid bilirubin dissolved in a $10^{-2} \mathrm{M}$ aqueous solution of sodium hydroxide was added to an aqueous solution of cupric chloride. A brownish-black precipitate was separated by centrifuging, and washed with distilled water, ethanol and chloroform, and then dried in vacuo into a solid substance (IX).

\section{Measurements}

In order to clarify the reaction of the bilirubin- $\mathrm{Cu}$ complex and to determine the optimum conditions for its production, spectrometric observation was performed with a Hitachi 139 type spectrophotometer and a Hitachi EPI-S type $_{2}$ infrared spectrophotometer.

\section{ResulTs}

\section{Conditions for the production of bilirubin-Cu complex}

Formation of bilirubin-Cu complex: In the first series of the experiment the visible spectrum of the solution III was investigated at various mole ratios of $\mathrm{Cu}$ to bilirubin in an equivolume mixture of ethanol and chloroform. Fig. 1 shows the spectra of the solution containing excess of Cu over bilirubin after standing for one hour at room temperature. The spectra $a, b$ and $c$ of this figure, corresponding to small excess of $\mathrm{Cu}$ (bilirubin: $\mathrm{Cu}=1: 1-6$ ), show two portions of absorption band on either side of an isosbestic point at about $500 \mathrm{~m} \mu$. Of these two absorptions, the one at $450 \mathrm{~m} \mu$ is a characteristic absorption of free bilirubin. 


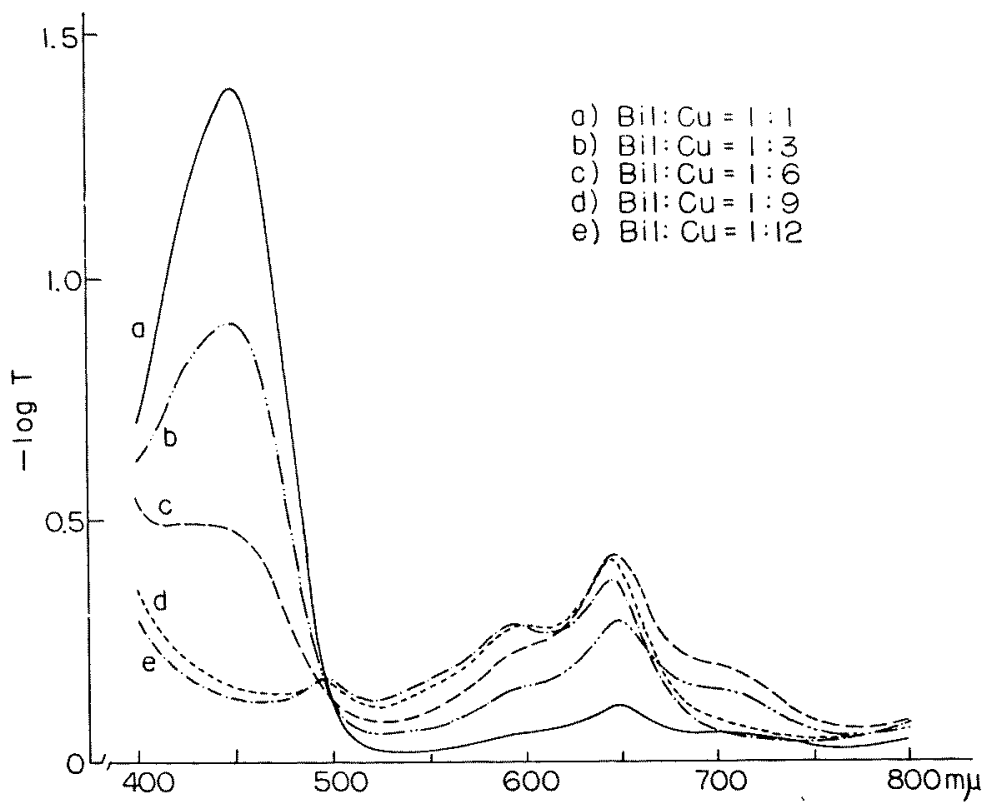

Fig. 1. Visible spectra of the solution III at various mole ratios of bilirubin vs. cupric chloride. Solvent, ethanol : chloroform $=1: 1$. One hour after mixture of the solutions I and II.

Therefore, the other one which is located at about $645 \mathrm{~m} \mu$ and associated with shoulder absorptions on both sides rperesents a newly formed complex compound. On the other hand, the spectra $d$ and $e$, corresponding to large excess of $\mathrm{Cu}$ (bilirubin: $\mathrm{Cu}=1: 9-12$ ), are devoid of the bilirubin absorption but show three distinct absorptions at 495, 595 and $645 \mathrm{~m} \mu$. This multiplicity of absorption suggests a possibility of secondary change of the complex in the presence of large excess $\mathrm{Cu}^{++}$. The next experiment, that is follow-up of the spectrum of the solution III, was carried out to evaluate this possibility.

Fig. 2 shows the changes of the visible spectrum of the solution III of bilirubin: $\mathrm{Cu}=1: 3$ when it was left standing at room temperature. An isosbestic point is seen at about $660 \mathrm{~m} \mu$. As time elapsed, the absorption in the range of longer wave length than this was weakened, and an absorption at $595 \mathrm{~m} \mu$, which was a mere shoulder of the $645 \mathrm{~m} \mu$ absorption in an initial phase of the reaction, gradually became a distinct absorption maximum. This change is interpreted as indicating maturation of the complex compound, i.e., transformation of a primary product into a more stable form. The result of a similar study at a larger excess of $\mathrm{Cu}$ (bilirubin: $\mathrm{Cu}=1: 9$ ) is shown in Fig. 3. In this case, the $645 \mathrm{~m} \mu$ absorption decreased and the $595 \mathrm{~m} \mu$ absorption increased in the intensity with the lapse of time, and a new absorption peak appeared at $495 \mathrm{~m} \mu$ one hour or more 


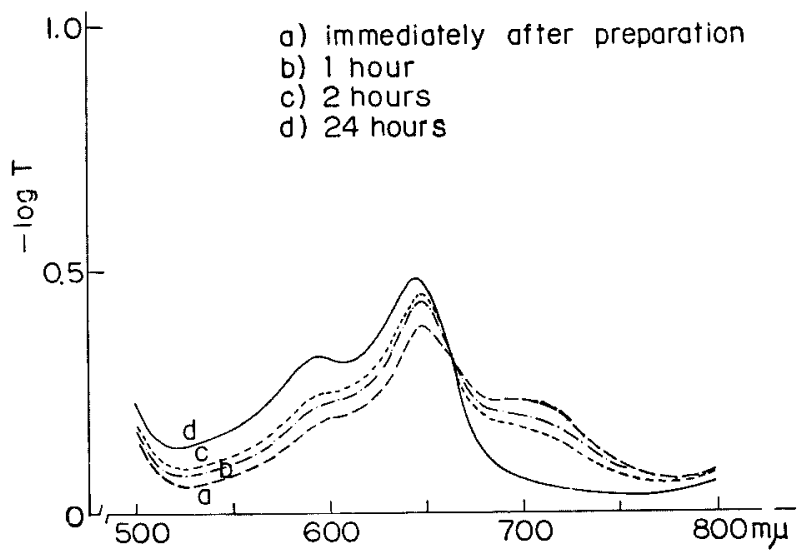

Fig. 2. Change of the spectrum of the solution III by time. Bilirubin: $\mathrm{Cu}=1: 3$. Solvent, ethanol : chloroform $=1: 1$.

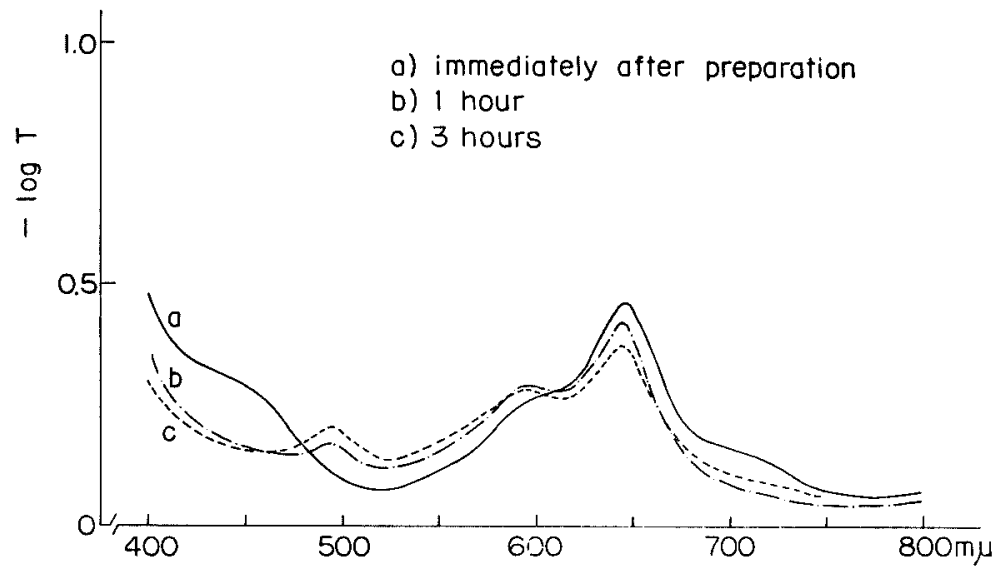

Fig. 3. Change of the spectrum of the solution III by time. Bilirubin : Cu=1:9. Solvent, ethanol : chloroform $=1: 1$.

after beginning of the reaction. It is concluded from these results that the stable bilirubin- $\mathrm{Cu}$ complex produced by the above-mentioned method is represented by absorptions at $595 \mathrm{~m} \mu$ and $645 \mathrm{~m} \mu$, and the absorption at $495 \mathrm{~m} \mu$ is due to oxidation product of this compound.

Fig. 4 is the result of another series of experiments in which the ratio of bilirubin to $\mathrm{Cu}$ was fixed at $1: 6$ and the composition of the solvent altered variously. The result suggests that ethanol accelerates the formation reaction of the complex. 


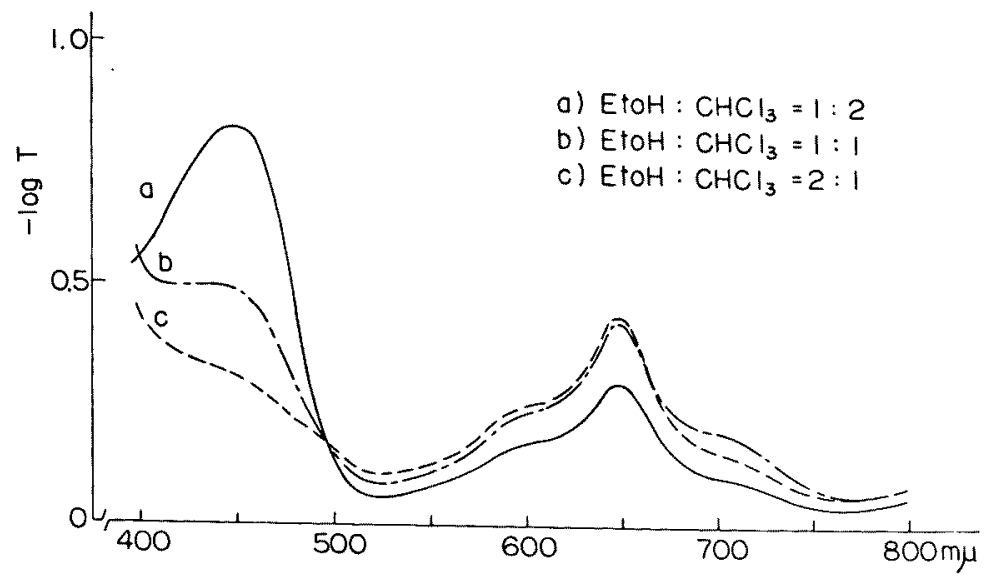

Fig. 4. Visible spectra of the solution III for various compositions of the solvent. Bilirubin : $\mathrm{Cu}=1: 6$. One hour after mixture of the solutions I and II.

Isolation of bilirubin-Cu complex: The above results show that the formation of the bilirubin- $\mathrm{Cu}$ complex proceeds effectively in the presence of a large excess of $\mathrm{Cu}^{++}$. But, excess $\mathrm{Cu}^{++}$causes a change of the product into the substance which shows a maximum absorption at $495 \mathrm{~m} \mu$. Therefore, in order to obtain the bilirubin- $\mathrm{Cu}$ complex, the excess $\mathrm{Cu}^{++}$has to be removed by washing with distilled

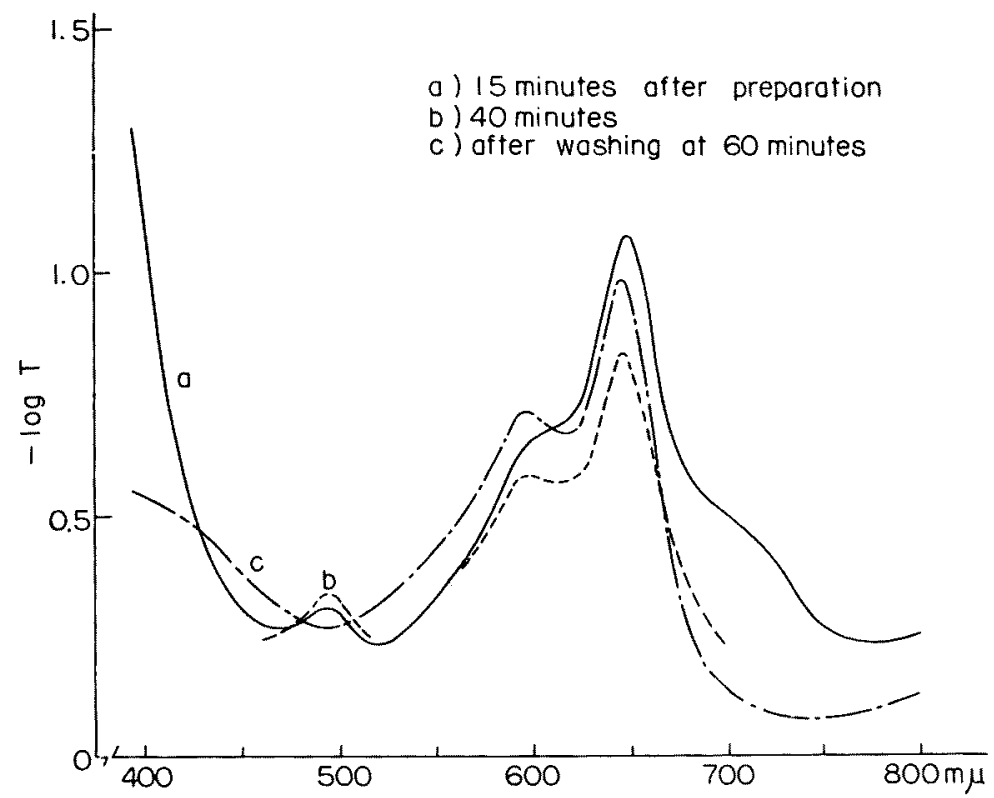

Fig. 5. The effect of well-timed washing of the solution III on its visible spectrum. Bilirubin : $\mathrm{Cu}=1: 40$. Solvent, ethanol : chloroform $=1: 1$. 
water after an appropriate period. The following experiments were performed to determine the optimum timing of the washing. Forty or 45 times excess of $\mathrm{Cu}^{++}$ was added to bilirubin in an equivolume mixture of ethanol and chloroform, and the mixture was followed up spectrometrically. When the solution. III of the above composition was thoroughly washed with distilled water after one hour's standing at room temperature, the absorption peak at $492 \mathrm{~m} \mu$ did not appear in the resulting solution IV (Fig. 5). But, when the washing was carried out after one and a half hours, that is after the absorption peak at $495 \mathrm{~m} \mu$ already became prominent, a new absorption peak at $525 \mathrm{~m} \mu$ appeared in the solution IV (Fig. 6).

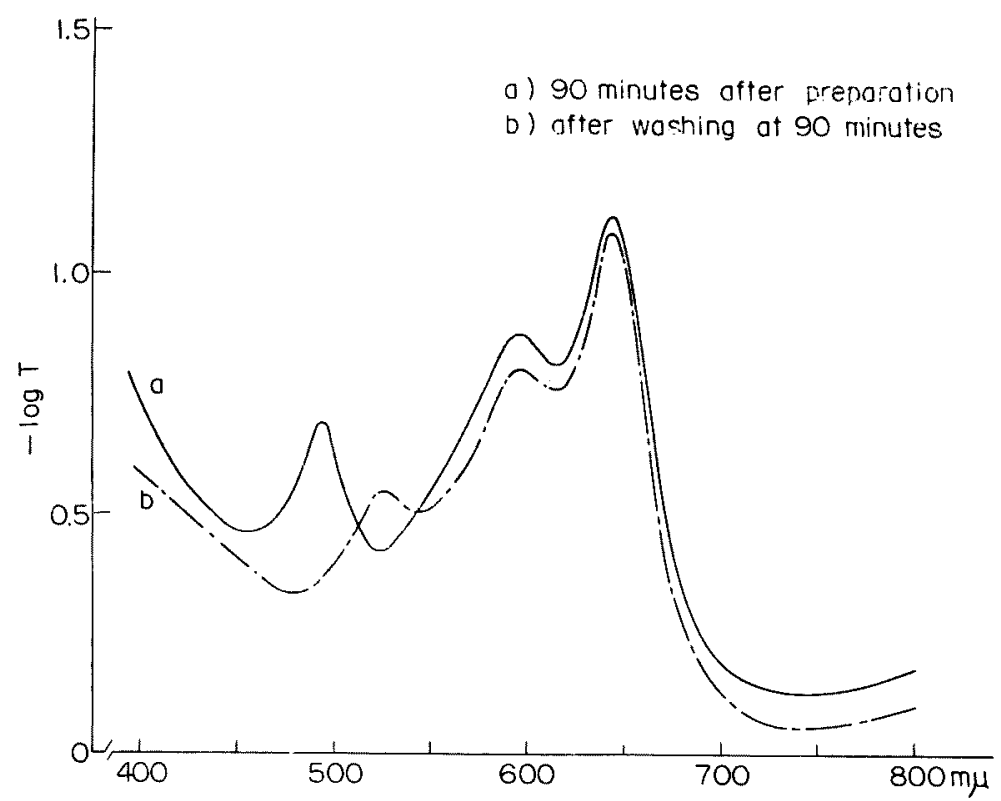

Fig. 6. The effect of delayed washing of the solution III on its visible spectrum. Bilirubin : $\mathrm{Cu}=1: 40$. Solvent, ethanol : chloroform $=1: 1$.

Accordingly, it was found that the best result was obtained when the solution III was washed after one hour's standing at room temperature. On each occasion, the washing of the solution III had a tint of purple with weak fluorescence, and was positive for the potassium ethylxanthogenate test.

The solution IV, freed from excess $\mathrm{Cu}^{++}$, was evaporated up in nitrogen flow, and the residue $\mathrm{V}$ was extracted with chloroform. Small absorption bands of $500-550 \mathrm{~m} \mu$ range disappeared with this procedure. The residue VI treated with chloroform was finally extracted with ethanol. The ultravioletvisible spectrum of the ethanol extract VII is shown in Fig. 7. In the visible region, this extract exhibited two distinct absorptions at $595 \mathrm{~m} \mu$ and $645 \mathrm{~m} \mu$, 


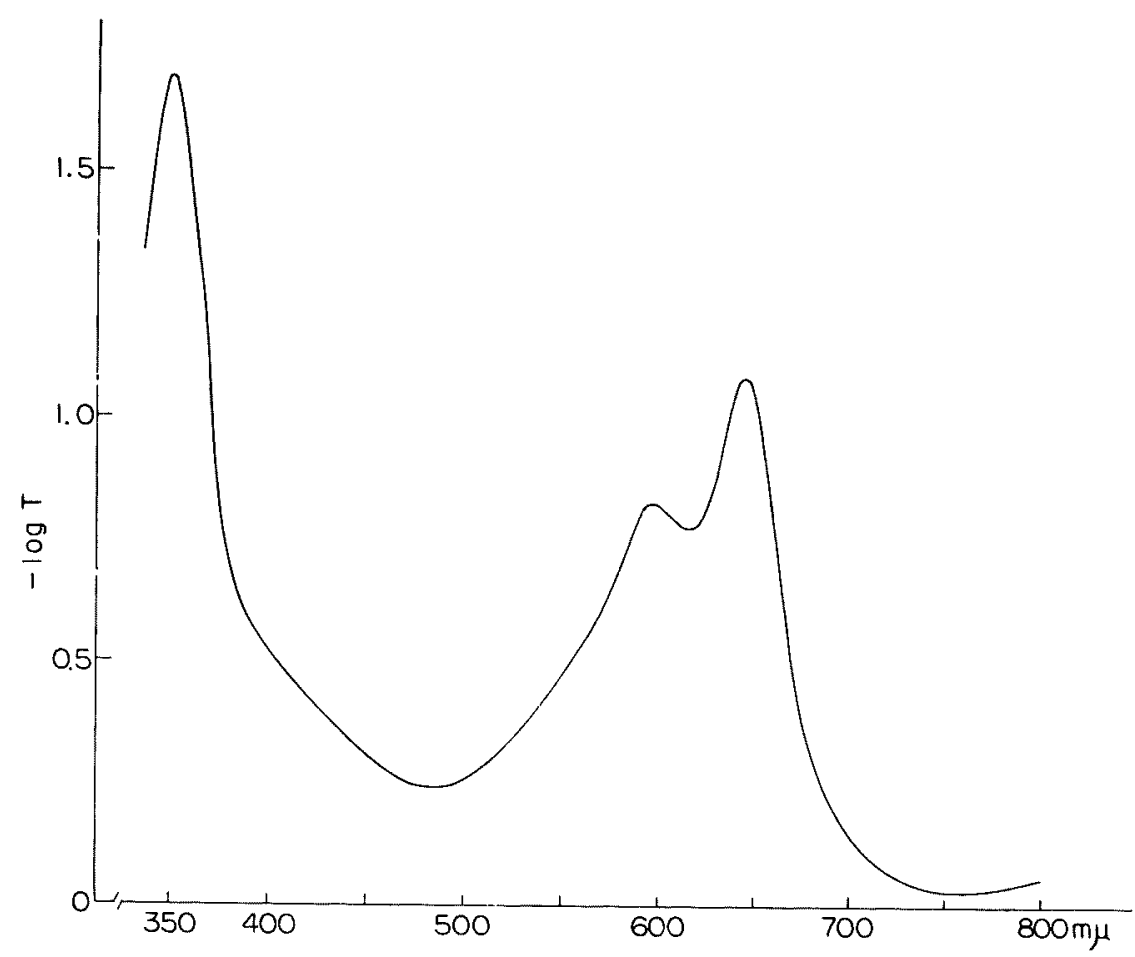

Fig. 7. Ultraviolet-visible spectrum of the sample VII.

exactly the same positions as the stable bilirubin-Cu complex identified in the crude solution III (Fig. 2). In the ultraviolet region, a very characteristic band was identifiable at $350 \mathrm{~m} \mu$.

\section{Infrared spectra of the products}

a) Bilirubin-Cu complex

The bilirubin-Cu complex recovered from the ethanol extract VII as the solid material (VIII) was lustrous powder of dark blue or black in color. The infrared spectrum of this substance measured by the $\mathrm{KBr}$-disk method is shown in Fig. 8 with that of free bilirubin for comparison. There were a number of differences between these two spectra, among which the following were noteworthy: (1) In free bilirubin, the absorption peak at $1,692 \mathrm{~cm}^{-1}$ was due to the stretching vibration of $\mathrm{C}=\mathrm{O}$ of carboxylic $-\mathrm{COOH}\left(\nu_{\mathrm{C}=0}\right)$, but in the bilirubin-Cu complex this shifted toward higher wave numbers, around $1,710 \mathrm{~cm}^{-1}$. (2) Free bilirubin did not show the $1,100 \mathrm{~cm}^{-1}$ band which was remarkable in the bilirubin-Cu complex. (3) The absorption band due to the stretching vibration of $\mathrm{N}-\mathrm{H}\left(\nu_{\mathrm{NH}}\right)$ at $3,400 \mathrm{~cm}^{-1}$ was much weaker in the bilirubin- $\mathrm{Cu}$ complex than in free bilirubin. 


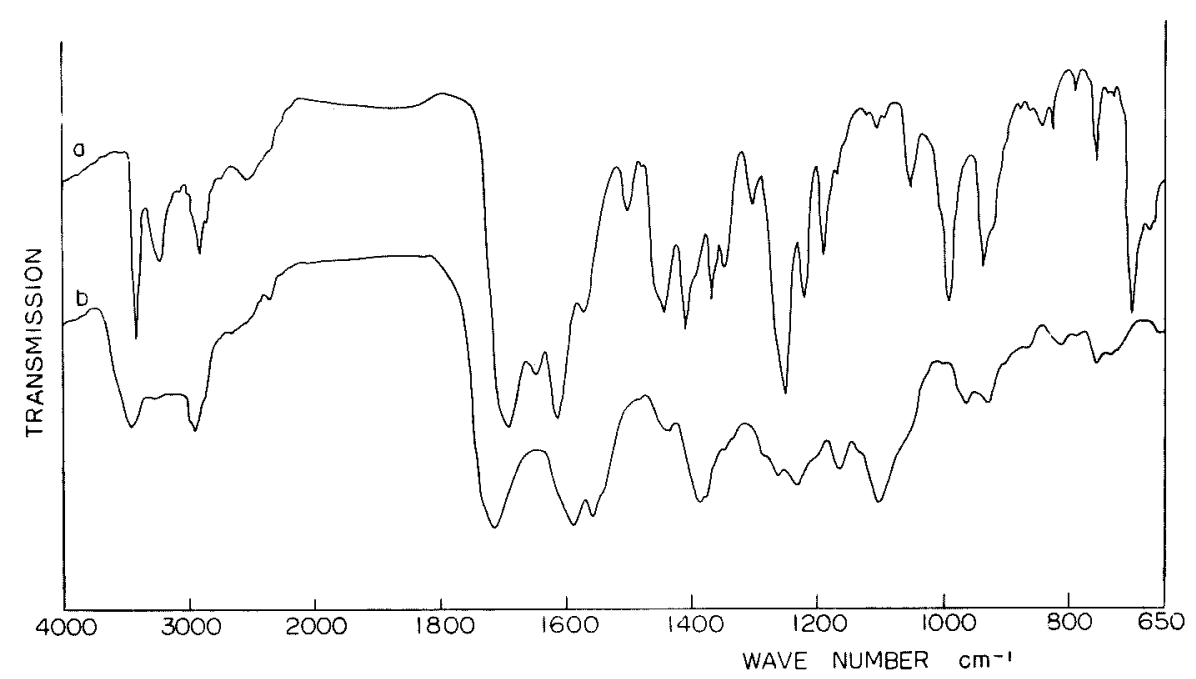

Fig. 8. Infrared spectra of free dibasic acid bilirubin (a) and the bilirubin-Cu complex (b). KBr-disk method.

\section{b) Bilirubin- $\mathrm{Na}-\mathrm{Cu}$ complex}

The bilirubin complex produced in aqueous solution in the presence of $\mathrm{Na}^{+}$ and $\mathrm{Cu}^{++}$(Sample IX) was non-lustrous black powder which was insoluble in water and organic solvents. The infrared spectrum of this substance was characterized by the absence of $\nu_{\mathrm{C}=0}$ of carboxylic acid and by the presence of strong and broad absorption bands at $1,560 \mathrm{~cm}^{-1}$ and $1,400 \mathrm{~cm}^{-1}$ which are attributable to $\nu_{\mathrm{C}=0}$ of carboxylate -COO-. However, the absorption due to $\nu_{\mathrm{C}=0}$ of free carboxylic acid appeared at $1,710 \mathrm{~cm}^{-1}$ when this substance was treated with dilute hydrochloric acid in a solvent mixture of ethanol and chloroform.

\section{Discussion}

It is known that macrocyclic tetrapyrroles, porphyrins and their analogous compounds have an intense absorption band at about $400 \mathrm{~m} \mu$ (the Soret band ${ }^{4,5}$ ), while bile pigments in which the conjugation of tetrapyrroles is interrupted lack this characteristic absorption. It is also known that the squareplanar divalent metal complexes of porphyrins generally have a Soret band and two visible bands, usually referred to as the $\alpha$ and $\beta$ bands. ${ }^{6}$ On the basis of such facts, the three absorption bands identified in the sample VII $(350,595$ and $645 \mathrm{~m} \mu)$ are presumed to correspond to the Soret band and to the $\alpha$ and $\beta$ bands of metalloporphyrins, respectively. This suggests that this substance has a metalloporphyrinlike structure in which the macrocycle may still be interrupted but a kind of ring configuration has been attained by coordination with $\mathrm{Cu}$. On the other hand, the infrared spectrum of this compound shows that the propionic acid groups attached 
to the pyrrole nuclei remain as free acid forms unlike in the case of the complex of bilirubin with calcium.? Therefore, $\mathrm{Cu}$ atom must be located in nitrogen atoms of two pyrroles as $\mathrm{N}-\mathrm{Cu}-\mathrm{N}$. Moreover, the appearance of a sharp absorption at $1,100 \mathrm{~cm}^{-1}$ and decrease of the $\nu_{\mathrm{NH}}$ absorption $\left(3,430 \mathrm{~cm}^{-1}\right)$ occurred in complex formation of bilirubin with $\mathrm{Cu}$. Since these phenomena are known to be specific to porphyrin derivatives, ${ }^{8,9}$ it is suggested that the sample VIII is a metalloporphyrinlike bilirubin-Cu complex. From these results it is clear that bilirubin, produced by oxidative cleavage of the $\alpha$-methine bridge $(-\mathrm{CH}=)$ of the porphyrin macrocycle, forms a ring structure by complex formation.

The present result disagrees with the opinion of Bentley ${ }^{10}$ that bilirubin, unlike biliverdin and mesobiliviolin, ${ }^{11,12}$ does not form a metal complex in spite of the presence of replaceable hydrogen atoms and nitrogen atoms available for coordination. He proposes the structure of a biliverdin-metal complex as shown in Fig. 9 in which one of the two terminal pyrrole nuclei is converted into the

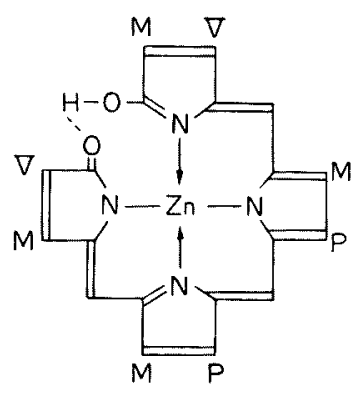

Fig. 9. Structure of a $\mathrm{Zn}$-complex of bilirubin (Bentley ${ }^{10}$ ).

lactam form and a stable hydrogen bond forms between the lactam carbonyl and the hydroxyl group of hydroxypyrrolenine. As an evidence for such a structure, it has been claimed that a methoxyl derivative of biliverdin (terminal hydroxyl groups substituted by $-\mathrm{OCH}_{3}$ ) does not form a metal complex. But, Fischer and his coworkers ${ }^{13}$ synthesized the $\mathrm{Zn}$-complexes of bilirubin and biliverdin derivatives in which hydroxyl groups had been replaced by methoxyl and the propionic acid groups esterified, Since the prototropy takes place in bilirubin (Fig. 10), ${ }^{14}$ it seems that this compound is able to form metalloporphyrin-like complexes.

In the synthesis of bilirubin-Cu complex in the presence of a great excess of $\mathrm{Cu}^{++}$, it was observed that the product $\left(\lambda_{\max }=595 \mathrm{~m} \mu\right.$ and $645 \mathrm{~m} \mu$ ) gradually changed to a purple substance $\left(\lambda_{\max }=495 \mathrm{~m} \mu\right)$. This is apparently due to oxidative effect of $\mathrm{Cu}^{++}$. However, the stability of the bilirubin-Cu complex was satisfactory in solutions containing a small excess of $\mathrm{Cu}^{++}$or after removal of excess $\mathrm{Cu}^{++}$by washing with water. Lemberg ${ }^{12}$ reported a similar phenomenon that a mesobiliverdin- $\mathrm{Cu}$ complex was oxidized into a mesobilivioilin-Cu complex 

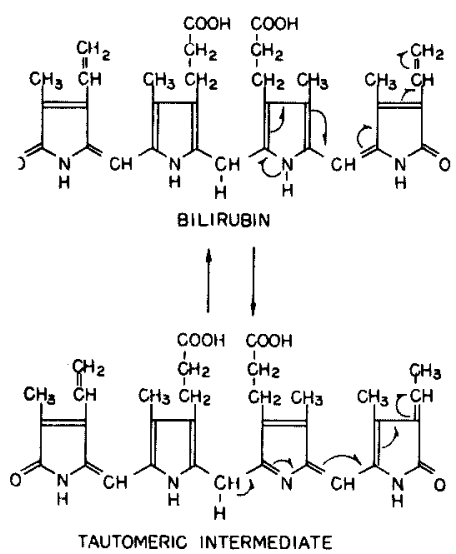

Fig. 10. Prototropy of bilirubin $\left(\right.$ Gray $\left.^{14}\right)$.

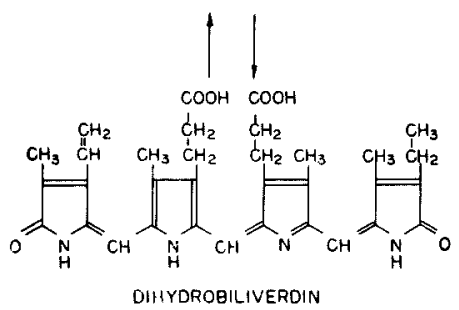

in the presence of excess $\mathrm{Cu}^{++}$.

The infrared spectrum of the other bilirubin complex, the sample IX, which was produced by reaction of cupric chloride with bilirubin dissolving in an aqueous solution containing sodium hydroxide, was different from that of the above-mentioned bilirubin-Cu complex. From the infrared spectrum of the sample IX, it is observed that unlike the latter complex or free bilirubin, this substance has carboxylate groups instead of free carboxylic groups. This suggests that $\mathrm{Cu}$ coordinates to nitrogen atoms of sodium bilirubinate.

The bilirubin- $\mathrm{Cu}$ complex and the bilirubin-Na-Cu complex prepared in vitro in this experiment are dark blue or black in color and quite resemble the pigments of genuine black colored gallstones. Of course, until identification of these complexes in the bile or gallstone, it remains obscure whether such bilirubin-metal complexes are produced in vivo and contribute toward black coloration of the gallstone. However, it is known that copper is a common component of bile ${ }^{3}$ and bile also includes other metal elements such as iron, magnesium and manganese, and these facts suggest the possibility of formation in vivo of metal complexes of bile pigments. Such possibilities are also supported by a number of reports which described the presence of bile pigments containing metal elements in vivo: for instance, a "green pigment" that is an iron containing tetrapyrrole compound as an intermediate product during transformation of hemin 
to bilirubin, ${ }^{12}$ and an unusual derivative of bilirubin associated with iron. ${ }^{15}$

\section{Acknowledgment}

The author thanks to Prof. T. Yamasaki and Prof. T. Kanno of the Institute of Metallurgy and Mineral Dressing, Tohoku University and to Prof. S. Ito of the Faculty of Science, Tohoku University for their encouragement and suggestions.

\section{References}

1) Suzuki, N. On black pigment of gallstones with special reference to difference from melanins. Tohoku J. exp. Med., 1965, 85, 238-247.

2) Suzuki, N. On black pigment of gallstones with special reference to comparison with bilirubin derivatives. Tohoku J. exp. Med., 1965, 85, 396-405.

3) Matsushiro, T., Nakamura, N. \& Takasawa, S. Copper content of bile as determined by x-ray fluorometry. Tohoku Igaku Zassi (Jap.), 1965, 71, 214-217.

4) Soret, J.L. Sur le spectre d'absorption du sang dans la partie violette et ultraviolette. C.R. Acad. Sci., 1883, 97, 1269-1270.

5) Gamgee, A. On the absorption of the extreme violet and ultra-violet rays of the spectrum by haemoglobin, its compounds and certain of its derivatives. Z. Biol., 1897, 34, 505-528.

6) Falk, J.E. Porphyrins and metalloporphyrins, Elsevier Publishing Co., New York, 1964.

7) Suzuki, N. \& Toyoda, M. On infrared absorption spectra of bilirubin and calcium bilirubinate. Tohoku J. exp. Med., 1966, 88, 353-360.

8) Mason, S.F. The infrared spectra of N-heteroaromatic systems; Part 1, the prophyrins. J. chem. Soc., 1958, pp. 976-982.

9) Weigl, J.W. \& Livingstone, R. Infrared spectra of chlorophyll and related compounds. J. Amer. chem. Soc., 1953, 75, 2173-2176.

10) Bentley, K.W. The chemistry of natural products, Vol. 5, Interscience Publishers, New York, 1960, pp. 162-163.

11) Lemberg, R. Bile pigments. VI. Biliverdin, uteroverdin and oocyan. Biochem. $J$,, $1934,28,978-987$.

12) Lemberg, R. Transformation of haemins into bile pigments. Biochem. J., 1935, 29, $1322-1336$.

13) Fischer, H., Plieninger, H. \& Weissbarth, O. Über die Konstitution des Bilirubins und über bilirubinoide Farbstoffe. Z. physiol. Chem., 1941, 268, 197-226.

14) Gray, C.H. Bile pigments in health and disease, Charles C. Thomas Publishers, Springfield, 1961, pp. 16-17.

15) Kosaka, A. On a variation of bilirubin. Tokyo I ji Shinshi (Jap.), 1949, 66, 573-577. 